\title{
Editorial to: Reading wide, writing wide in the digital age
}

\author{
Miriam Llamas Ubieto ${ }^{1}$
}

Published online: 20 March 2017

(C) Akadémiai Kiadó, Budapest, Hungary 2017

The special issue "Reading Wide, Writing Wide in the Digital Age" can be classified under the theoretical studies on digital literature and culture that, within Digital Humanities, take a qualitative and critical approach to their object of study. They deal with the production, circulation and reception of electronic textualities, widening the more restrictive notion what can be considered a literary text, while consciously considering the epistemological models that may be productive in their analysis. The selected papers presented here attempt to give a variety of responses to an increasingly important issue: how the 'wide' aspect linked to digital environments affects literary and textual practices and what the consequences may be.

The particular characteristics of the digital medium have contributed to an unprecedented acceleration in the circulation of materials, texts, memories, knowledge and subjects, as well as a proliferation of interconnections on a huge, even global scale, while the paradigm of the national and the local still coexists alongside it. Changes to communicative processes and the way knowledge is accessed involve a broader, more dynamic and interconnected view of the world, a change in perception.

The spread and increase of digital archives, the expansive forms of the globalisation of knowledge, the agglutinative extension of textual information and multimedia are breaking through barriers and allowing the dissemination of the object, be it text, book, corpus or subject.

This leads us to wonder, firstly, given the wide, transcultural and even global scope of digital technology, if textual, particularly literary, practices of production, circulation and reception, are really changing; if this circulation is in fact changing writing and ways of reading. It is important to reflect on whether this circulation is widening literary practices by means of new forms of distribution, new communities

Miriam Llamas Ubieto

mllamasu@ucm.es

1 Madrid, Spain 
of writers and readers with their own rituals, new codes, new imaginaries or new ways of positioning themselves in relation to the previous and the local. Secondly, because of the ability of digital technology to expand, multiply and widen both the material and the archive, we wonder if it is also widening writing and reading practices and pushing the boundaries of the device, the book, the author, the conventional or academic act of reading, that is, a crossover that is more than a mere extrapolation of previous traditions.

A double process of peer review at two different stages, once as abstracts and then as fully written up papers, resulted in the six papers included here. They all coincide that digital literary studies need to be "widened" to study the intersection between what we consider as the "digital turn" and the "spatial turn" of our era. In fact, in the same way that we argue that the practice of reading can be affected by the 'widening' effect of the digital, the same happens with approaches to studying both texts and literary practices in the digital era: although academic, they are also readings and are subject to that 'widening'. All the authors we present, from their various academic origins, have found that the spatial concepts and metaphors that we call the "spatial turn" can be productive epistemological models to approach the problem. This spatialisation by means of 'vectors', 'crossovers', 'syntopies', circulations, fields, maps and ubiquities is not only due to the imaginary and conceptual metaphor suggested by the term 'wide', but also to the impact of spatial technology development in shaping knowledge and humanistic disciplines. However, unlike the static use of spatial conceptualisation to explain the digital culture that goes back further than the metaphor of the net, all the papers point to a dynamism of space, or in space, which breaks boundaries and explains the novel writing and reading phenomena found.

The papers selected, when examining these processes of change from different angles, offer a fairly comprehensive all-round view. Papers by Odile Farge, Amelia Sanz and Germán Sierra address the new practices of production or writing in the digital space and the forms that these practices take on by being part of circulation on a global scale, as well as the new global communities and imaginaries. Odile Farge in "Authoring Software-tools in Digital Literatures: A Vector of a Global Imaginary" asks how globalised software is changing writing. She defines it as a tool for constructing a discursive global imaginary that becomes a vector that moulds new writing practices. A new global code mediates the possibilities and the boundaries of individual authorship and influences its writing and artistic creation practices, whereby writers are connected to globalisation processes. "Digital Literatures Circulation: Testing Post-Bourdieu Theories", by Amelia Sanz, uses post-Bourdieu methodology to explore the particular case of Digital Literatures that were born to circulate on the globalised internet, to investigate the consequences of that circulation and the emergence of a dynamic field not encapsulated by national boundaries but that responds to new forms of institutionalisation at the global level. Germán Sierra tackles the new global codes and formulas and new international communities of writers in "Postdigital Synchronicity and Syntopy: The Manipulation of Universal Codes" and also shows how the new forms taken on by literary products break through the boundaries of the literary object itself. Practices and 
products adopt codes and aesthetics to adapt to the global, the synchronic and the syntopic and are also a sign of a postdigital art.

The issue of both productive and receptive widening literary practices is precisely the focus of the paper by Ana Marques da Silva and Sandra Bettencourt entitled "Writing-Reading Devices: Crossovers". Through their detailed microanalyses they show the postdigital transgression of writing boundaries, the act of reading and of the book as device, explaining how these elements widen in praxis by means of intersections, crossovers and intermediations leading to a distributed authorship and reimaginations of genres. Diogo Marques in "Poetic Fingerprints: Digital Literature's Countercultural and Metamedial Integration of Vision and Touch" explores also these transgressions dealing with the integration of sense modalities such a touch and haptic visuality.

Lastly, paper by Enrique Santos is focused on the transformation and widening of reading practices. In "GIS and Telescopic Reading: between Spatial and Digital Humanities" Santos deals with the change in paradigm of literary reading in the academic sphere that has occurred with the arrival of GIS in the digital and cartographic turn. Santos puts forward a wide study of literature using what he terms a telescopic reading that interlinks quantitative and qualitative methods.

In all cases, the study of wide circulation in digital environments enables us to highlight novelty and change in reading and writing practices in the digital era. 